\title{
An Equivalent Propellant Sloshing Model of Ellipsoid Cylindrical Tank
}

\author{
Tengda Xin ${ }^{1,2, a}$, Yongsheng Duan ${ }^{1,2, b}$, Shuangbang Zhang ${ }^{1, c}$, Cunyan Cui ${ }^{2, d}$, Guoyu Bai ${ }^{3, \text { e }}$ \\ ${ }^{1}$ Key Laboratory of Space Launching Site Reliability Technology, Haikou, China \\ ${ }^{2}$ Space Engineering University, Beijing, China \\ ${ }^{3}$ Jiuquan Satellite Launch Center, Jiuquan, China \\ axtd0701@126.com, \\ bduanys_vip@yeah.net, czhangbs74@163.com, ${ }^{\mathrm{d}} 13661315668 @ 126 . c o m,{ }^{\mathrm{e}}$ guoyu50@yeah.net
}

Keywords: Equivalent model; Propellant sloshing; Ellipsoid cylindrical tank; Liquid rocket

\begin{abstract}
The propellant sloshing is a significant factor for the design of rocket structure and control system. In order to solve the propellant sloshing problems in ellipsoid cylindrical tank, an equivalent model is established based on the sloshing model of cylindrical tank. The equivalent modeling criterions are proposed according to the sloshing characteristic parameters. And then the equivalent formulas are deduced based on the ellipsoid cylindrical tank model and the criterions. The equivalent model is verified through comparing with the test sloshing parameters. The results show that the differences of sloshing parameters are less than $5 \%$, so that the equivalent model can provide a reference for the design of the rocket.
\end{abstract}

\section{Introduction}

The propellant tank is a fatal part of the liquid rocket, and is not merely used to store the liquid propellant that accounts for about $90 \%$ of the launch weight, but the main bearing structure of the liquid rocket. The propellant sloshing in the tank can seriously affects the reliability and stability of the rocket. In 1969, the landing accuracy of the Apollo 11 is affected, because of the remaining propellant caused an unexpected wobble[1]. In 1998, the mission to detect the asteroid was delayed by 13 months, according to the coupling between the NEAR's motion and the propellant sloshing[2]. In 2007, the second stage of Falcon 1 rocket went out of control in flight, and the sloshing of propellant was blamed for the accident[3]. The propellant sloshing in the tank is widely studied by the aerospace researchers, and achieved fruitful achievements[4][5]. However, the researches are mainly focused on the simple structure tanks, because of the boundary conditions of these tanks can easily be confirmed, such as the cylindrical tank[6], the rectangular tank[7] and the spherical tank[8].

In engineering applications, the ellipsoid cylindrical propellant tank is the main type tank to liquid rocket. In this paper, an equivalent propellant sloshing model of ellipsoid cylindrical tank is established to provide a reference for the design of rocket structure and control system.

\section{Sloshing Model of Cylindrical Tank}

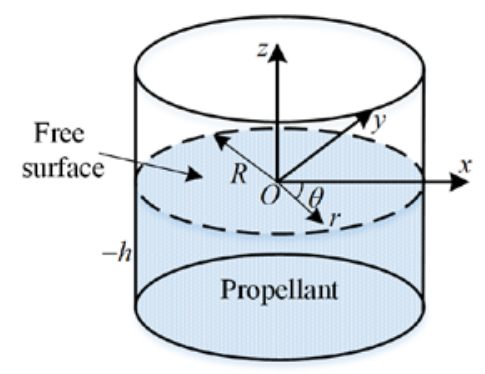

Fig. 1 The cylindrical tank model 
This chapter is to analysis the sloshing characteristics to provide the foundation to establish the equivalent model. The propellant sloshing in cylindrical tank (Fig. 1) is widely studied by researchers, and is one of the few sloshing problems with analytical solutions[9].

In Fig. $1, R$ is the radius of cylindrical tank, $h$ is the height of propellant in cylindrical tank, Oxyz is the Cartesian coordinate, and $\operatorname{Or} \theta z$ is the cylindrical coordinate.

Based on the fluid field equations of tank, the Laplace equation and the boundary conditions can be expressed as

$$
\begin{aligned}
& \nabla^{2} \tilde{\Phi}=0 \\
& \partial \tilde{\Phi} / \partial r=0, \text { at } r=R \\
& \partial \tilde{\Phi} / \partial z=0, \text { at } z=-h
\end{aligned}
$$

The general solution of Eq.(1) subject to the boundary conditions Eq.(2) and Eq.(3) is

$$
\tilde{\Phi}(r, \theta, z, r)=\sum_{m=0}^{\infty} \sum_{n=1}^{\infty}\left[\alpha_{m n}(t) \cos m \theta+\beta_{m n}(t) \sin m \theta\right] J_{m}\left(\lambda_{m n} r\right) \frac{\cosh \left[\lambda_{m n}(z+h)\right]}{\cosh \lambda_{m n} h}
$$

In Eq. 4, $\tilde{\Phi}$ is the velocity potential function, $\alpha_{m n}$ and $\beta_{m n}$ are time dependent to be determined from the free-surface initial conditions, $m$ and $n$ are the radial and circular modes, $\lambda_{m n}=\xi_{m n} / R$ are the roots of $\partial J_{m}\left(\lambda_{m n} r\right) / \partial r=0$ at $r=R, J_{m}(\cdot)$ is the Bessel function. When $R=1 \mathrm{~m}, m$ and $n$ take different values respectively, the sample inherent mode of each model as shown in Fig. 2.
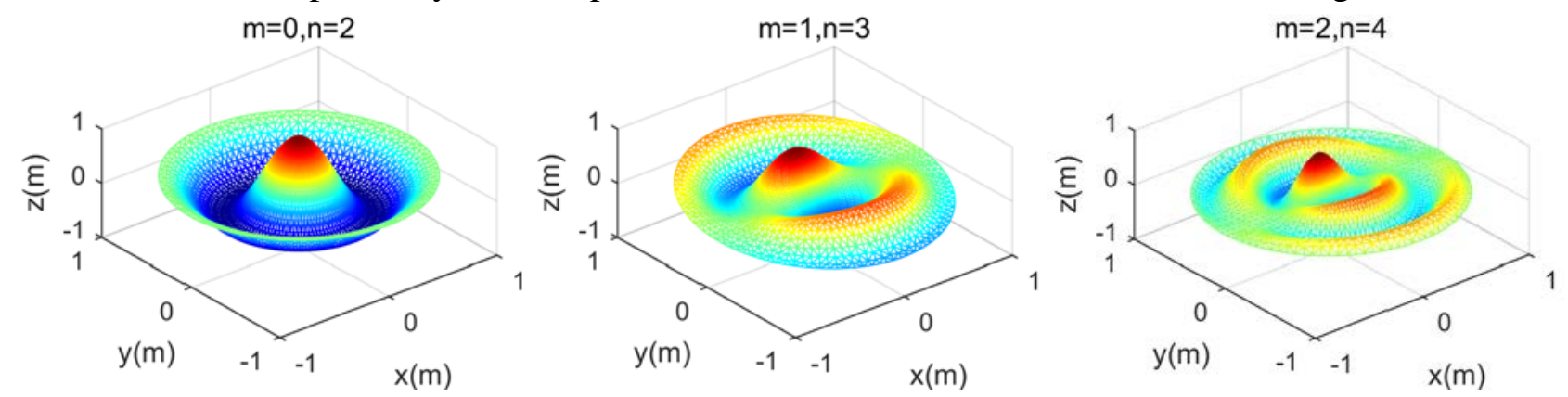

Fig. 2 The sample inherent mode

If $\alpha_{m n}$ and $\beta_{m n}$ is expressed as $\sin \omega_{m n} t$, the inherent oscillation frequency of fluid free surface can be obtained

$$
\omega_{m n}^{2}=g \xi_{m n} \tanh \left(\xi_{m n} h / R\right) / R
$$

Because $\tanh \left(\xi_{m n} h / R\right)$ is a monotone increasing function in the range of $(-\infty,+\infty)$, when $\left(\xi_{m n} h / R\right) \geq 3, \tanh \left(\xi_{m n} h / R\right) \approx 1$. So when $\lambda_{m n} h \geq 3$, Eq. 5 can be expressed as

$$
\omega_{m n}^{2}=g \xi_{m n} / R
$$

The fluid surface elevation $\eta$ measured from the undisturbed free surface is obtained

$$
\eta=\frac{1}{g} \sum_{m=0}^{\infty} \sum_{n=1}^{\infty}\left[\alpha_{m n} \cos m \theta+\beta_{m n} \sin m \theta\right] J_{m}\left(\lambda_{m n} r\right) \cosh \left(\lambda_{m n} h\right)\left(\omega_{m n} \cos \omega_{m n} t\right)
$$

Consider the cylindrical tank under sinusoidal excitation along the $x$ axis

$$
X(t)=X_{0} \sin \Omega t
$$


In Eq. 8, $X_{0}$ and $\Omega$ are the excitation amplitude and frequency, respectively. The hydrodynamic pressure $p$ at any point due to propellant sloshing (neglecting the hydrostatic pressure $\rho g h$ ) can be expressed as

$$
p=\rho X_{0} \Omega^{2} \cos \theta \cos \Omega t \times\left\{r+\sum_{n=1}^{\infty} \frac{2 R}{\left(\xi_{1 n}^{2}-1\right)} \frac{\Omega^{2}}{\left(\omega_{1 n}^{2}-\Omega^{2}\right)} \frac{J_{1}\left(\xi_{1 n} r / R\right)}{J_{1}\left(\xi_{1 n}\right)} \frac{\cosh \left[\xi_{1 n}(z+h) / R\right]}{\cosh \xi_{1 n} h / R}\right\}
$$

where $\rho$ is the density of propellant.

\section{Equivalent Model of Ellipsoid Cylindrical Tank}

According to Eq.4, Eq.5, Eq.7 and Eq.9, we know that the sloshing characteristics of propellant have a considerable relationship with the tank radius $R$ and the height of propellant $h$. As the propellant height increases the relationship between the sloshing characteristic and the shape of tank bottom becomes smaller and smaller. Hence, the tank radius is the most important factor to be considered, and the next is the height of propellant.

In order to establish the equivalent propellant sloshing model of ellipsoid cylindrical tank, the modeling criterions proposed in this paper are as follows

1) The free surface area of the propellant in the ellipsoid cylindrical tank is the same as that in the cylindrical tank, so as to determine the equivalent radius.

2) The propellant volume in the ellipsoid cylindrical tank is the same as that in the cylindrical tank, so as to determine the equivalent height of propellant.

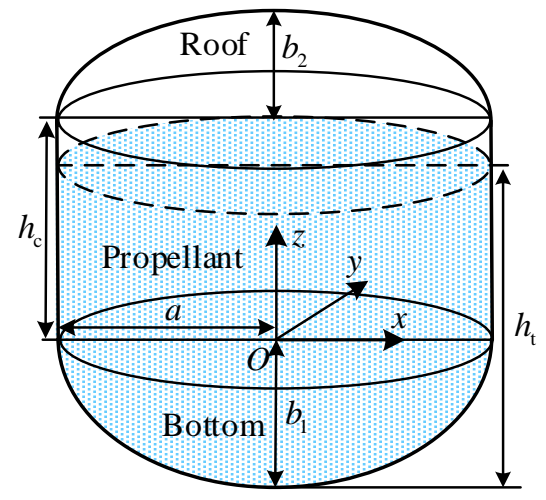

Fig. 3 The ellipsoid cylindrical tank model

In Fig. 3, $a$ is the horizontal semi-axis of ellipsoid cylindrical tank, $b_{1}$ is the semi-axis of tank bottom, $b_{2}$ is the vertical semi-axis of tank roof, $h_{\mathrm{t}}$ is the height of propellant in ellipsoid cylindrical tank, and $h_{\mathrm{c}}$ is the cylinder height of ellipsoid cylindrical tank.

According to Fig. 3, we can obtain the elliptic equation of tank bottom generatrix

$x^{2} / a^{2}+z^{2} / b_{1}^{2}=1, \quad-a \leq x \leq a$

And the elliptic equation of tank roof generatrix is

$x^{2} / a^{2}+\left(z-h_{\mathrm{c}}\right)^{2} / b_{1}^{2}=1, \quad-a \leq x \leq a$

Using Eq. 10 and Eq. 11, the equivalent radius $R_{\mathrm{e}}$ can be obtained

$$
R_{\mathrm{e}}= \begin{cases}a \sqrt{2 h_{\mathrm{t}} / b_{1}-h_{\mathrm{t}}^{2} / b_{1}^{2}} & 0 \leq h_{\mathrm{t}}<b_{1} \\ a & b_{1} \leq h_{\mathrm{t}}<b_{1}+h_{c} \\ a \sqrt{1-\left(h_{\mathrm{t}}-h_{\mathrm{c}}-b_{1}\right)^{2} / b_{2}{ }^{2}} & b_{1}+h_{c} \leq h_{\mathrm{t}}<h_{c}+b_{1}+b_{2}\end{cases}
$$


The propellant volume $V$ in the ellipsoid cylindrical tank can be expressed as

$$
V= \begin{cases}2 \pi a^{2} b_{1} / 3-\pi a^{2}\left(b_{1}-h_{\mathrm{t}}\right)+\pi a^{2}\left(b_{1}-h_{\mathrm{t}}\right)^{3} / 3 b_{1}^{2} & 0 \leq h_{\mathrm{t}}<b_{1} \\ 2 \pi a^{2} b_{1} / 3+\pi a^{2}\left(h_{\mathrm{t}}-b_{1}\right) & b_{1} \leq h_{\mathrm{t}}<b_{1}+h_{c} \\ \pi a^{2}\left[h_{\mathrm{t}}-b_{1} / 3-\left(h_{\mathrm{t}}-h_{\mathrm{c}}-b_{1}\right)^{3} / 3 b_{2}^{2}\right] & b_{1}+h_{c} \leq h_{\mathrm{t}}<h_{c}+b_{1}+b_{2}\end{cases}
$$

Using Eq. 12 and Eq. 13, the equivalent height of propellant $h_{\mathrm{e}}$ can be obtained

$$
h_{\mathrm{e}}= \begin{cases}\left(3 b_{1} h_{\mathrm{t}}-h_{\mathrm{t}}^{2}\right) /\left(6 b_{1}-3 h_{\mathrm{t}}\right) & 0 \leq h_{\mathrm{t}}<b_{1} \\ h_{\mathrm{t}}-b_{1} / 3 & b_{1} \leq h_{\mathrm{t}}<b_{1}+h_{c} \\ {\left[3 b_{2}^{2} h_{\mathrm{t}}-b_{2}^{2} b_{1}-\left(h_{\mathrm{t}}-h_{\mathrm{c}}-b_{1}\right)^{3}\right] /\left[3 b_{2}^{2}-3\left(h_{\mathrm{t}}-h_{\mathrm{c}}-b_{1}\right)^{2}\right]} & b_{1}+h_{c} \leq h_{\mathrm{t}}<h_{c}+b_{1}+b_{2}\end{cases}
$$

Therefore, according to Eq. 12 and Eq. 14, the sloshing model of ellipsoid cylindrical tank can be transformed into the sloshing model of an equivalent cylindrical tank.

For example, a certain ellipsoid cylindrical tank, $b_{1}=0.5 \mathrm{~m}, b_{2}=0.5 \mathrm{~m}, h_{\mathrm{c}}=1 \mathrm{~m}$. Using Eq. 12 and Eq. 14, the variations of equivalent radius and equivalent height of propellant can be obtained as shown in Fig. 4.
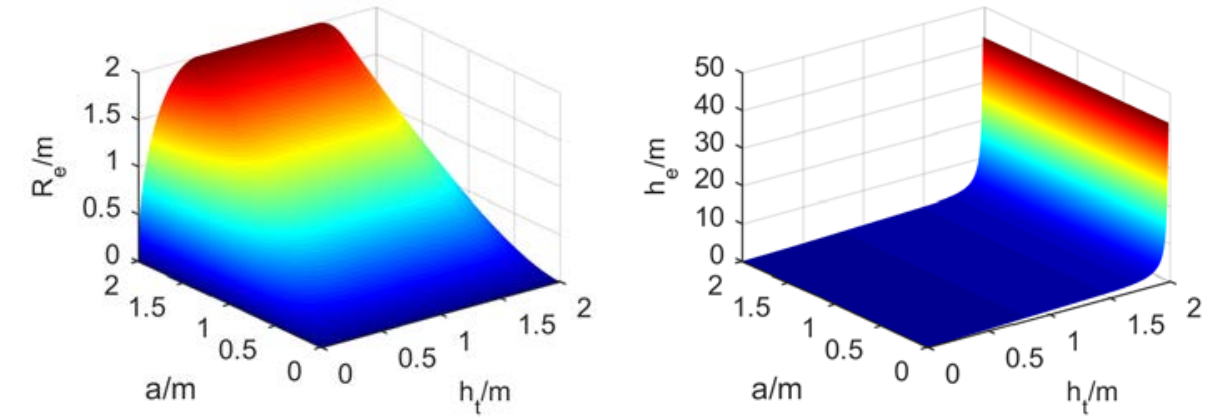

Fig. 4 Equivalent radius and equivalent height of propellant

Fig. 4 shows that, $R_{\mathrm{e}}$ increases with the increase of $a$, and when $0 \leq h_{\mathrm{t}}<b_{1} R_{\mathrm{e}}$ increases with the increase of $h_{\mathrm{t}}$, when $b_{1} \leq h_{\mathrm{t}}<b_{1}+h \quad R_{\mathrm{e}}$ holds constant, and when $b_{1}+h_{c} \leq h_{\mathrm{t}}<h_{c}+b_{1}+b_{2} \quad R_{\mathrm{e}}$ decreases with the increase of $h_{\mathrm{t}}$. The $a$ has no effect on $h_{\mathrm{e}}$, and $h_{\mathrm{e}}$ increases with the increase of $h_{\mathrm{t}}$, especially when $b_{1}+h_{c} \leq h_{\mathrm{t}}<h_{c}+b_{1}+b_{2}, h_{\mathrm{e}}$ increases rapidly.

\section{Equivalent Model Verification}

In this paper, we take the test data of Ares 1 US LOX tank[10] for example to verify the equivalent model. The parameters of the ellipsoid tank in the test are $b_{1}=0.99 R, b_{2}=0.57 R, h_{\mathrm{c}}=0.71 R$, and $a=R$.

According to the pendulum model of propellant sloshing, for a cylindrical tank the sloshing frequency $f_{\mathrm{s}}$ can be expressed as

$$
f_{\mathrm{s}}=\frac{1}{2 \pi} \sqrt{1.841 \frac{g}{R} \tanh \left(1.841 \frac{h}{R}\right)}
$$

where $g$ is the acceleration of gravity.

And the sloshing mass $m_{\mathrm{s}}$ can be expressed as 


$$
m_{\mathrm{s}}=m \frac{R}{2.199 h} \tanh \left(1.841 \frac{h}{R}\right)
$$

where $m$ is the total propellant mass.

Using the Eq. 12, Eq. 14, Eq. 15 and Eq. 16. The sloshing frequency and sloshing mass can be obtained, as shown in Table 1.

Table 1 The equivalent and test sloshing frequency

\begin{tabular}{cccccc}
\hline$h_{\mathrm{t}} / R$ & $h_{\mathrm{e}} / R_{\mathrm{e}}$ & $2 \pi \sqrt{R / g} f_{\mathrm{es}}$ & $2 \pi \sqrt{R / g} f_{\mathrm{ts}}$ & $m_{\mathrm{es}} / m$ & $m_{\mathrm{ts}} / m$ \\
\hline 0.769 & 0.478 & 1.140 & 1.160 & 0.672 & 0.701 \\
0.966 & 0.637 & 1.232 & 1.240 & 0.589 & 0.562 \\
1.180 & 0.850 & 1.299 & 1.300 & 0.490 & 0.512 \\
1.320 & 0.990 & 1.322 & 1.330 & 0.436 & 0.439 \\
1.470 & 1.140 & 1.337 & 1.340 & 0.387 & 0.389 \\
1.670 & 1.340 & 1.347 & 1.350 & $0.335(0.3345)$ & 0.335 \\
1.770 & 1.473 & 1.351 & 1.390 & 0.306 & 0.292 \\
\hline
\end{tabular}

In Table $1, f_{\mathrm{es}}$ is the equivalent sloshing frequency, $f_{\mathrm{ts}}$ is the test sloshing frequency, $m_{\mathrm{es}}$ is the equivalent sloshing mass, and $m_{\mathrm{ts}}$ is the test sloshing mass.

Hence, according to the results as shown in Table 1 . The differences of the sloshing frequency and sloshing mass can be obtained, as shown in Fig. 5.

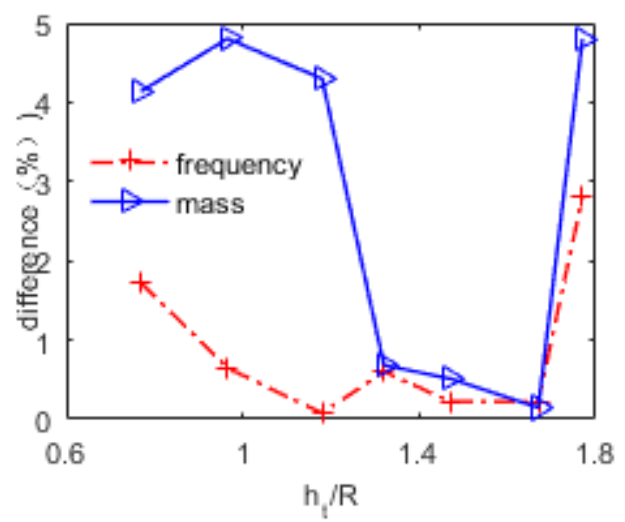

Fig. 5 The difference of sloshing frequency and sloshing mass

Fig. 5 shows that, in general the difference of sloshing mass is less than $5 \%$. And the differences of sloshing frequency is less than $3 \%$, especially when $b_{1} \leq h_{\mathrm{t}}<b_{1}+h$, the difference of sloshing frequency are less than $1 \%$. The differences of sloshing frequency and sloshing mass trend to decrease and then increase with the increase of $h_{\mathrm{t}} / R$.

\section{Summary}

This paper established an equivalent propellant sloshing model of ellipsoid cylindrical tank based on the sloshing model of cylindrical tank. And the effectiveness of equivalent propellant sloshing model is verified through comparing with the test sloshing frequency and sloshing mass of an ellipsoid cylindrical tank. The results shows that, the differences of sloshing frequency and sloshing mass are less than $5 \%$ in general, and when $b_{1} \leq h_{\mathrm{t}}<b_{1}+h$ the difference of frequency is less than $1 \%$. Therefore, in engineering application the equivalent propellant tank can provide a reference for the design of rocket structure and control system. 


\section{Acknowledgement}

The authors gratefully acknowledge the financial support on this work from the Key Laboratory of Space Launching Site Reliability Technology of China.

\section{References}

[1] D.A. Mindell. Digital Apollo: Human and Machine in Spaceflight [M]. The MIT Press, 2008: 181-217.

[2] E.J. Hoffman, C.J. Gay, W.L. Ebert. Final report of the NEAR anomaly review board [R]. 1999: $1-15$.

[3] B. Berger. Space X declares Falcon 1 rocket operational despite less than perfect test [N] Space.com. 2007.

[4] H. N. Abramson. The dynamic behavior of liquids in moving containers [R]. NASA SP-106, 1967: 1-10.

[5] D. M. Eggleston. Dynamic stability of space vehicles volume xiv-testing for booster propellant sloshing parameters [R]. NASA CR-948, 2018: 5-23.

[6] D. L. Cui, S. Z. Yan, X. S. Guo, et al. Parametric resonance of liquid sloshing in partially filled spacecraft tanks during the powered-flight phase of rocket [J]. Aerospace Science and Technology, 2014, 35: 93-105.

[7] O. M. Faltinsen, A. N. Timokha. Asymptotic modal approximation of nonlinear resonant sloshing in a rectangular tank with small fluid depth [J]. Journal of Fluid Mechanics, 2002, 470: 319-357.

[8] P. Mciver. Sloshing frequencies for cylindrical and spherical containers filled to an arbitrary depth [J]. Journal of Fluid Mechanics, 1989, 201: 243-257.

[9] R.A. Ibrahim. Liquid sloshing dynamics theory and applications [M]. New York: Cambridge University Press, 2005: 88-95.

[10] J.G. Perez, R.A. Parks, D.R. Lazor. Validation of slosh model parameters and anti-slosh baffle designs of propellant tanks by using lateral slosh testing [C]. $27^{\text {th }}$ Aerospace Testing Seminar, 2012. 